\title{
Fluorotetrahydroquinolines from Diethyl 2-Fluoromalonate Ester
}

\author{
Craig A. Fisher ${ }^{\mathrm{a}}$, Antal Harsanyia ${ }^{\mathrm{a}}$ Graham Sandford ${ }^{\star a}$, Dmitri S. Yufit ${ }^{\mathrm{b}}$, and Judith A. K. Howard ${ }^{\mathrm{b}}$
}

\begin{abstract}
A short series of fluorotetrahydroquinolines was synthesised in two steps from diethyl fluoromalonate and appropriate ortho-nitrobenzyl bromide precursors.
\end{abstract}

Keywords: Fluorinated heterocycle · Fluoromalonate · Fluorotetrahydroquinoline · Organofluorine chemistry · Selective fluorination

\section{Introduction}

The development of effective methods for the synthesis of selectively fluorinated heterocyclic systems continues to grow in importance due to the increasing number of commercially significant fluorinated heterocyclic structural units that are present in valuable pharmaceuticals and agrochemicals. ${ }^{11]}$ While fluoroheteroaromatic systems are sub-structures of many drugs, there are comparatively very few instances where saturated fluorinated heterocyclic rings form part of an active pharmaceutical ingredient (API) or marketed drug structure. ${ }^{[1]}$

As part of a long-term programme exploring the synthesis of fluorinated organic systems using elemental fluorine ${ }^{[2]}$ for the crucial synthetic step of carbonfluorine bond formation, we have established efficient methods for the large scale preparation of fluorinated 1,3-dicarbonyl derivatives including 1,3-ketoesters ${ }^{[3]}$ and malonate systems. ${ }^{[4]}$ Whereas the application of non-fluorinated malonate esters to the synthesis of a wide range of structurally sophisticated target molecules is, of course, well documented in general

\section{Results and Discussion}

Our strategy for the synthesis of fluorotetrahydroquinolines from 2-diethyl 2-fluoromalonate ester $\mathbf{1}$ is outlined in Scheme 1 in which, in principle, reaction of fluoromalonate with ortho-nitrobenzyl bromide derivatives followed by reduction-cyclisation would lead to the fluorotetrahydroquinoline targets.

Reaction of ortho-nitrobenzyl bromide derivatives 2 with anions formed by deprotonation of diethyl 2-fluoromalonate ester 1 using sodium hydride in DMF gave good yields (unoptimised) of diester derivatives 3 which were purified by column chromatography on silica gel (Table 1). The molecular structure of diester $\mathbf{3 c}$ was confirmed by X-ray crystallography (Fig.1).

By a similar process, reaction of the analogous mesylate system $\mathbf{4}$ with two equivalents of $\mathbf{1}$ gave the fluorinated diester 3e and significant quantities of a tetraester derivative $\mathbf{5}$ by a mechanism shown in Scheme 2. Dehydrofluorination of $\mathbf{3 e}$ to an intermediate alkene which then reacts with a further equivalent of $\mathbf{1}$ by a Michael addition process gives $\mathbf{5}$ in good yield. The structure of $\mathbf{5}$ was confirmed by X-ray crystallography (Fig. 2) and contains two virtually identical independent molecules linked together by a number of C-H...O interactions. The relatively short (2.934 $\AA$ ) intermolecular F...Cl contacts may be regarded as halogen bonds. ${ }^{[9]}$

Reductions of the nitro groups in fluorodiester substrates 3 and subsequent cyclisation to fluorotetrahydroquinolines 6 were accomplished by using either sodium dithionite or hydrogenation over a palladium on carbon catalyst (Table 2). The molecular structure of fluorotetrahydroquinoline 6a was confirmed by X-ray crystallography (Fig. 3).

The conformation of the stereogenic centre in $\mathbf{6 a}$ is unusual in that the smaller fluorine atom occupies the equatorial position while the larger ester functionality is axial. $\alpha$-Fluoroamides have a very strong preference (syn-anti $7.5 \mathrm{kcal} \mathrm{mol}^{-1}$ ) for the carbon-fluorine bond to adopt an anti-pla-

\footnotetext{
${ }^{*}$ Correspondence: Prof. G. Sandford E-mail: Graham.Sandford@durham.ac.uk aDepartment of Chemistry Durham University, South Road Durham, DH1 3LE, UK ${ }^{\mathrm{b}}$ Chemical Crystallography Durham University
}

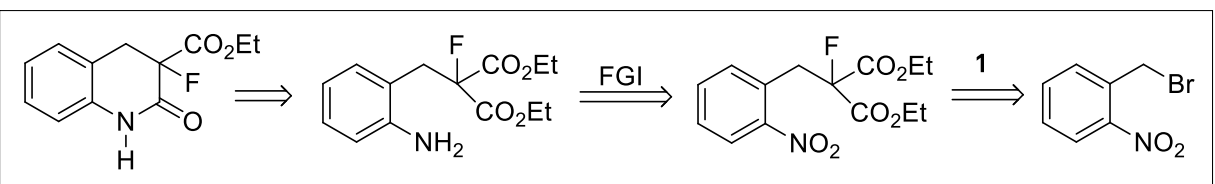

Scheme 1. Synthetic strategy for the preparation of fluorotetrahydroquinoline derivatives. 
Table 1. Synthesis of diesters 3<smiles>CCOC(=O)C(F)C(=O)OCC</smiles><smiles>O=[N+]([O-])c1c[Y]#ccc1CBr</smiles>

2

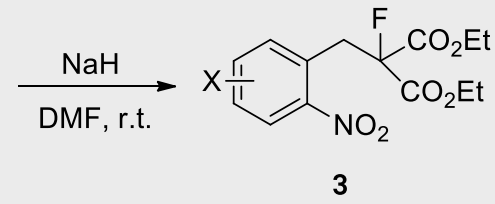

Diester 3<smiles>[B]Cc1ccccc1[N+](=O)[O-]</smiles>
$2 a$<smiles>CCOC(=O)C(F)(Cc1ccccc1[N+](=O)[O-])C(=O)OCC</smiles><smiles>N#Cc1ccc(CBr)c([N+](=O)[O-])c1</smiles><smiles>CCOC(=O)C(F)(Cc1ccc(C#N)cc1[N+](=O)[O-])C(=O)OCC</smiles>
3b, $54 \%$<smiles>COc1ccc(CBr)c([N+](=O)[O-])c1</smiles><smiles>CCOC(=O)C(F)(Cc1ccc(OC)cc1[N+](=O)[O-])C(=O)OCC</smiles>
$3 c, 56 \%$<smiles>O=[N+]([O-])c1cccc(F)c1CBr</smiles>

2d<smiles>CCOC(=O)C(F)(Cc1c(F)cccc1[N+](=O)[O-])C(=O)OCC</smiles>

\section{Experimental}

\subsection{General}

Proton, fluorine and carbon nuclear magnetic resonance (NMR) spectra $\left({ }^{1} \mathrm{H}\right.$, ${ }^{19} \mathrm{~F}$ and ${ }^{13} \mathrm{C}$ NMR) were obtained from a Bruker 400 Ultrashield spectrometer $\left({ }^{1} \mathrm{H}\right.$ $\mathrm{NMR}$ at $400 \mathrm{MHz},{ }^{19} \mathrm{~F}$ NMR at $376 \mathrm{MHz}$ and ${ }^{13} \mathrm{C}$ NMR at $101 \mathrm{MHz}$ ) using residual solvent peaks as the internal standard $\left({ }^{1} \mathrm{H} \mathrm{NMR} ; \mathrm{CHCl}_{3}\right.$ at $7.26 \mathrm{ppm},{ }^{19} \mathrm{~F} \mathrm{NMR}$; $\mathrm{CFCl}_{3}$ at $0.00 \mathrm{ppm}$ and ${ }^{13} \mathrm{C} \mathrm{NMR} ; \mathrm{CDCl}_{3}$ at $77.16 \mathrm{ppm})$. NMR spectroscopic data are reported as follows: chemical shift (ppm), integration, multiplicity $(\mathrm{s}=$ singlet, $\mathrm{d}=$ doublet, $\mathrm{t}=$ triplet, $\mathrm{q}=$ quartet, $\mathrm{m}=$ multiplet), coupling constant (Hz) and assignment.

GC-MS data were obtained from a Trace GC-MS device (Thermo-Finnigan Corporation) operating in electron impact ionization (EI) mode. Accurate mass analysis was performed on a Xevo QtoF mass spectrometer (Waters Ltd, UK) with an atmospheric solids analysis probe (ASAP). Melting point data was obtained using a Gallenkamp apparatus at atmospheric pressure and are uncorrected. Infra-red

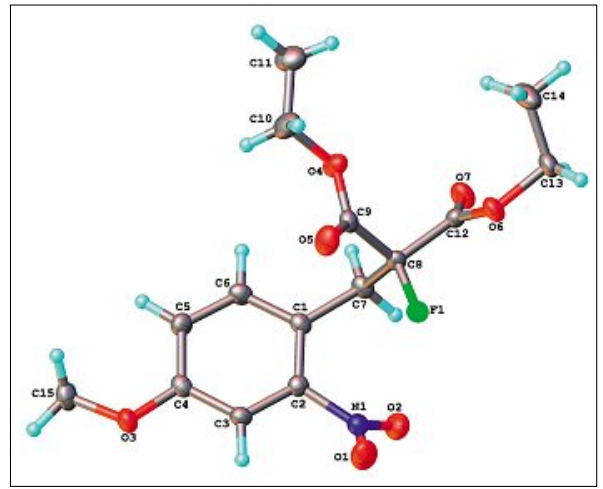

Fig. 1. Molecular structure of 3c.

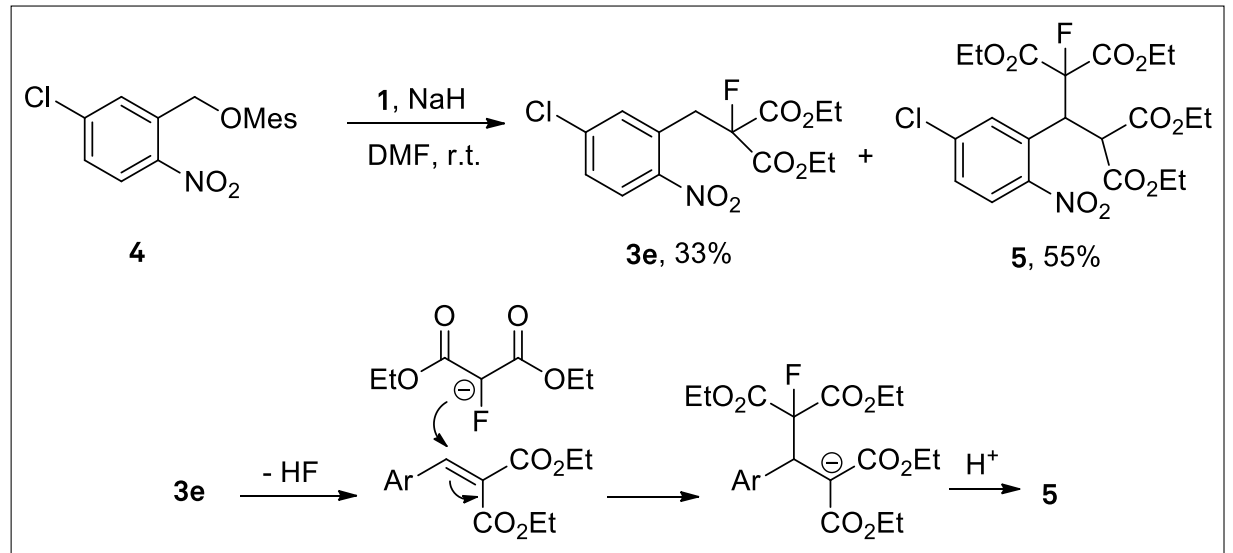

Scheme 2. Reaction of mesylate derivative $\mathbf{4}$ with $\mathbf{1 .}$

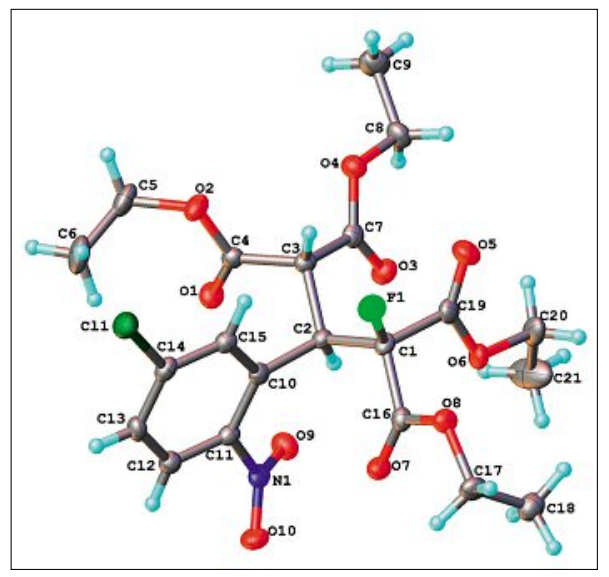

Fig. 2. Molecular structure of one of two independent molecules of 5 .
(IR) spectroscopy was performed on a Perkin Elmer 1600 Series FTIR with an ATR probe.

Single crystal X-ray data were collected at $120.0 \mathrm{~K}$ on a Bruker SMART CCD $1 \mathrm{~K}$ (compounds $\mathbf{3 c}$ and $\mathbf{6 a}$ ) and an Agilent XCalibur (compound 5) diffractometers ( $\lambda$ MoK $\alpha$, graphite monochromators, $\lambda=0.71073 \AA, \omega$-scan) equipped with Cryostream (Oxford Cryosystems) openflow nitrogen cryostats. All structures were solved by direct methods and refined by full-matrix least squares on $\mathrm{F}^{2}$ for all data using SHELXTL software. ${ }^{[11]}$ All non-hydrogen atoms were refined with anisotropic displacement parameters, $\mathrm{H}$-atoms in the structures $\mathbf{3 c}$ and $\mathbf{6 a}$ were located on the difference map and refined isotropically. The $\mathrm{H}$ atoms in twinned structure in the solid-state structure of fluorotetrahydroquinoline $6 \mathbf{a}$. 
Table 2. Synthesis of fluorotetrahydroquinolines 6 by reduction-cyclisation processes.

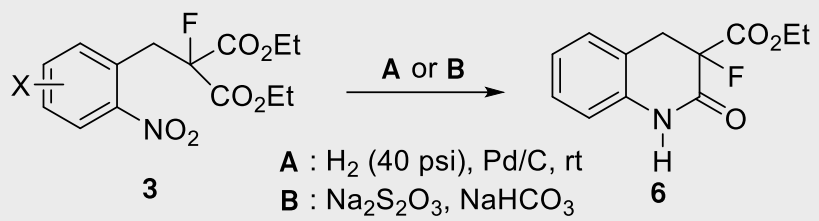

Diester 3 Fluorotetrahydoquinoline 6
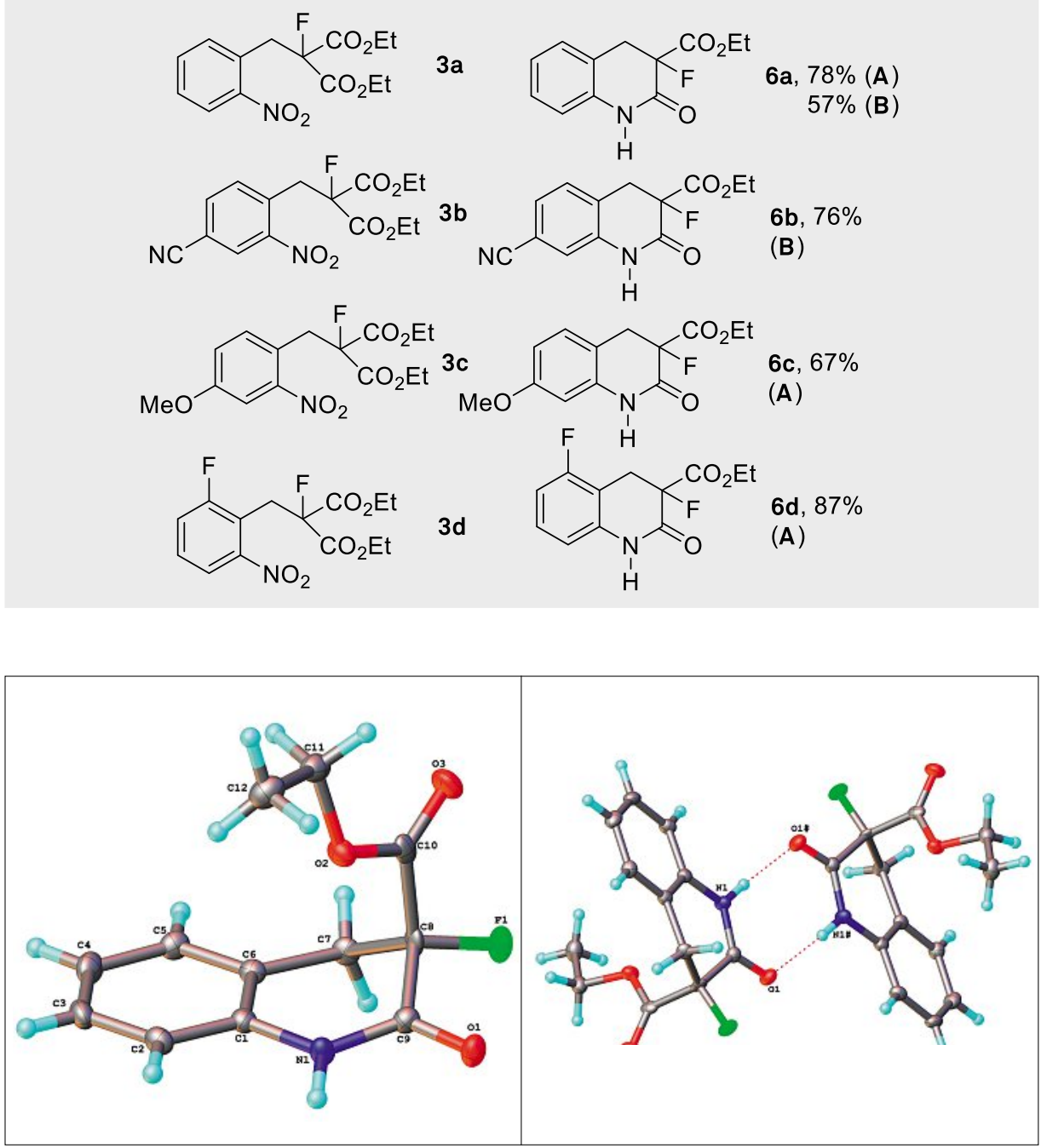

Fig. 3. Molecule $\mathbf{6 a}$ (left) and the $\mathrm{H}$-bonded dimer in the structure of $\mathbf{6 a}$ (right).

5 were placed in calculated positions and refined in riding mode. Crystallographic data for the structures have been deposited with the Chembridge Crystallographic Data Centre as supplementary publications CCDC 991436-991438.

Crystal Data for $3 c: \mathrm{C}_{15} \mathrm{H}_{18} \mathrm{NO}_{7} \mathrm{~F}, M=$ 343.30, monoclinic, space group $\mathrm{P} 2 / \mathrm{c}, a=$ $8.5269(7), b=11.8392(10), c=16.4098(14)$ $\AA$, $\beta=98.129(2)^{\circ}, V=1640.0(2) \AA^{3}, Z=4$, $T=120.0 \mathrm{~K}, \mu(\mathrm{MoK} \alpha)=0.118 \mathrm{~mm}^{-1}, D_{\text {cal }}$ $=1.390 \mathrm{~g} / \mathrm{mm}^{3}, 22040$ reflections measured $(4.26 \leq 2 \theta \leq 58), 4365$ unique $\left(R_{\text {int }}=\right.$ $0.0427)$ were used in all calculations. The final $R_{1}=0.0444(3279>2 \sigma(\mathrm{I}))$ and $w R_{2}=$ 0.1133 (all data), GOOF $=1.010$.

Crystal Data for 5: $\mathrm{C}_{21} \mathrm{H}_{25} \mathrm{ClFNO}_{10}$, $M=505.87$, monoclinic, space group $\mathrm{P} 2_{1} / \mathrm{c}, a=25.4199(12), b=9.5099(3)$, $c=21.6317(11) \AA, \beta=114.838(6)^{\circ}$, $V=4745.5(4) \AA^{3}, Z=8, T=120.0 \mathrm{~K}$, $\mu(\mathrm{MoK} \alpha)=0.224 \mathrm{~mm}^{-1}, D_{\text {calc }}=1.416 \mathrm{~g} /$ $\mathrm{mm}^{3}$, 52581 reflections measured (5.14 $\leq 2 \theta \leq 56), 11455$ unique $\left(R_{\text {int }}=0.1057\right)$ were used in all calculations. The final $R_{1}$ $=0.0633(7562>2 \sigma(\mathrm{I}))$ and $w R_{2}=0.1730$ (all data), $\mathrm{GOOF}=1.033$.

Crystal Data for 6a: $\mathrm{C}_{12} \mathrm{H}_{12} \mathrm{NO}_{3} \mathrm{~F}$ $(M=237.23)$ : monoclinic, space group $\mathrm{P} 2 / \mathrm{c}, a=10.5275(12), b=6.6687(8), c$ $=15.6871(18) \AA, \beta=102.677(2)^{\circ}, V=$ $1074.5(2) \AA^{3}, Z=4, T=120.0 \mathrm{~K}, \mu(\mathrm{MoK} \alpha)$ $=0.117 \mathrm{~mm}^{-1}, D_{\text {calc }}=1.466 \mathrm{~g} / \mathrm{mm}^{3}, 9083$ reflections measured $(3.96 \leq 2 \theta \leq 58)$, 2841 unique $\left(R_{\text {int }}=0.0684\right)$ were used in all calculations. The final $R_{1}=0.0643$ $(1827>2 \sigma(\mathrm{I}))$ and $w R_{2}=0.1601$ (all data), $\mathrm{GOOF}=1.020$.

\subsection{Synthesis of Fluorodiesters 3 4.2.1 General Procedure}

Sodium hydride $(60 \% \mathrm{NaH}$ in mineral oil) was washed with hexane $(2 \times 50$ $\mathrm{mL})$ and added to DMF (15 mL). Diethyl 2-fluoromalonate 1 in DMF (10 mL) and the 2-nitrobenzyl bromide derivative $\mathbf{2}$ in DMF $(15 \mathrm{~mL})$ were added dropwise to the solution which was stirred at room temperature for $19 \mathrm{~h}$. The reaction mixture was added to ice/water $(150 \mathrm{~mL})$, extracted with diethyl ether $(3 \times 50 \mathrm{~mL})$, washed sequentially with water $(3 \times 50 \mathrm{~mL})$ and brine $(3 \times 50 \mathrm{~mL})$, dried $\left(\mathrm{MgSO}_{4}\right)$, concentrated and purified by column chromatography on silica gel or recrystallization to give the pure fluorodiester $\mathbf{3}$.

\subsubsection{Diethyl 2-fluoro-2-(2-nitro- benzyl)malonate (3a)}

Sodium hydride $(0.31 \mathrm{~g}, 13.11 \mathrm{mmol})$, 1 (2.00 g, $11.24 \mathrm{mmol})$ and 2-nitrobenzyl bromide 2a (2.11 g, $9.79 \mathrm{mmol})$, after recrystallization, gave diethyl 2-fluoro2-(2-nitrobenzyl)malonate 3a (2.67 g, 87 $\%)$ as pale yellow crystals; mp $51-52{ }^{\circ} \mathrm{C}$; $\left([\mathrm{MH}]^{+}, 314.1053 . \mathrm{C}_{14} \mathrm{H}_{16} \mathrm{NO}_{6} \mathrm{~F}\right.$ requires $\left.[\mathrm{MH}]^{+}, 314.1040\right) ; v_{\max }\left(\right.$ neat, $\left.\mathrm{cm}^{-1}\right) 2989$, 1748, 1523, 1347, 1245, 1190, 1142, 1060; ${ }^{1} \mathrm{H}$ NMR $\delta 7.88\left(1 \mathrm{H}, \mathrm{dd},{ }^{3} J_{\mathrm{HH}} 8.0,{ }^{4} J_{\mathrm{HH}} 1.2\right.$, Ar-H), 7.57-7.39 (3H, m, Ar-H), 4.37-4.14 $\left(4 \mathrm{H}, \mathrm{m}, \mathrm{OCH}_{2}\right), 3.95\left(2 \mathrm{H}, \mathrm{d},{ }^{3} \mathrm{~J}_{\mathrm{HF}} 23.5, \mathrm{CF}-\right.$ $\left.\mathrm{CH}_{2}\right), 1.25\left(6 \mathrm{H}, \mathrm{t},{ }^{3} \mathrm{~J}_{\mathrm{HH}} 7.1, \mathrm{CH}_{3}\right) ;{ }^{19} \mathrm{~F}$ NMR $\delta-164.06\left(\mathrm{t},{ }^{3} J_{\mathrm{HF}} 23.5\right) ;{ }^{13} \mathrm{C}$ NMR $\delta 165.40$ $\left(\mathrm{d},{ }^{2} J_{\mathrm{CF}} 25.5, \mathrm{C}=\mathrm{O}\right), 150.74\left(\mathrm{~s}, \mathrm{C}-\mathrm{NO}_{2}\right)$, 133.34 (d, $\left.{ }^{3} J_{\mathrm{CF}} 2.4, \mathrm{C}-1\right), 132.72$ (s, Ar), 128.82 (s, Ar), 127.76 (s, Ar), 125.02 (s, Ar), 93.90 (d, ${ }^{1} J_{\mathrm{CF}}$ 201.8, C-F), 63.10 (s, $\left.\mathrm{OCH}_{2}\right), 35.36\left(\mathrm{~d},{ }^{2} J_{\mathrm{CF}} 20.3, \mathrm{CF}-\mathrm{CH}_{2}\right), 13.97$ $\left(\mathrm{s}, \mathrm{CH}_{3}\right) ; \mathrm{m} / z(\mathrm{ASAP}) 314.1\left([\mathrm{MH}]^{+}, 94 \%\right)$.

\subsubsection{Diethyl 2-(4-cyano-2-nitro- benzyl)-2-fluoromalonate (3b)}

Sodium hydride $(0.10 \mathrm{~g}, 4.32 \mathrm{mmol})$, 1 (0.62 g, $3.49 \mathrm{mmol}$ ) and 4-(bromomethyl)-3-nitrobenzonitrile $\mathbf{2 b}$ (0.80 g, $3.32 \mathrm{mmol}$ ), after column chromatography and recrystallization, gave diethyl 2-(4-cyano-2-nitrobenzyl)-2-fluoromalonate $\mathbf{3 b}$ $(0.61 \mathrm{~g}, 54 \%)$ as a yellow solid; mp 52-53 ${ }^{\circ} \mathrm{C}$; $\left([\mathrm{MH}]^{+}, 339.1036 . \mathrm{C}_{15} \mathrm{H}_{15} \mathrm{~N}_{2} \mathrm{O}_{6} \mathrm{~F}\right.$ requires $\left.[\mathrm{MH}]^{+}, 339.0992\right) ; v_{\max }\left(\right.$ neat, $\mathrm{cm}^{-1}$ ) 2982, 2234, 1763, 1541, 1279, 1239, 1196, 1131,$1060 ;{ }^{1} \mathrm{H}$ NMR $\delta 8.19(1 \mathrm{H}, \mathrm{s}, \mathrm{H}-3)$, $7.82\left(1 \mathrm{H}, \mathrm{dd},{ }^{3} J_{\mathrm{HH}} 8.1,{ }^{4} J_{\mathrm{HH}} 1.5, \mathrm{H}-5\right), 7.64$ $\left(1 \mathrm{H}, \mathrm{d},{ }^{3} \mathrm{~J}_{\mathrm{HH}} 8.1, \mathrm{H}-6\right), 4.31-4.18(4 \mathrm{H}, \mathrm{m}$, $\left.\mathrm{OCH}_{2}\right), 3.98\left(2 \mathrm{H}, \mathrm{d},{ }^{3} J_{\mathrm{HF}} 22.5, \mathrm{CF}-\mathrm{CH}_{2}\right)$, $1.27\left(6 \mathrm{H}, \mathrm{t},{ }^{3} \mathrm{~J}_{\mathrm{HH}} 7.1, \mathrm{CH}_{3}\right) ;{ }^{19} \mathrm{~F} \mathrm{NMR} \delta$ $-163.64\left(\mathrm{t},{ }^{3} J_{\mathrm{HF}} 22.5\right) ;{ }^{13} \mathrm{C}$ NMR $\delta 163.86$ $\left(\mathrm{d},{ }^{2} J_{\mathrm{CF}} 25.3, \mathrm{C}=\mathrm{O}\right), 149.59\left(\mathrm{~s}, \mathrm{C}-\mathrm{NO}_{2}\right)$, 134.36 (s, Ar), 133.62 (d, $\left.{ }^{3} J_{\mathrm{CF}} 2.7, \mathrm{C}-1\right)$, 132.02 (s, Ar), 127.46 (s, Ar), 115.25 (s, C-4), 112.25 (s, CN), 92.22 (d, ${ }^{1} J_{\mathrm{CF}} 202.8$, $\mathrm{CF}), 62.34\left(\mathrm{OCH}_{2}\right), 34.28\left(\mathrm{~d},{ }^{2} J_{\mathrm{CF}} 20.1\right.$, $\left.\mathrm{CF}-\mathrm{CH}_{2}\right), 12.87\left(\mathrm{~s}, \mathrm{CH}_{3}\right) ; \mathrm{m} / \mathrm{z}$ (ASAP) $339.1\left([\mathrm{MH}]^{+}, 100 \%\right), 311.1(25), 265.1$ (21), 173.0 (44). 


\subsubsection{Diethyl 2-fluoro-2-(4-methoxy-} 2-nitrobenzyl)malonate (3c)

Sodium hydride $(0.10 \mathrm{~g}, 4.23 \mathrm{mmol})$, 1 (0.61 g, $3.42 \mathrm{mmol}$ ) and 1-(bromomethyl)-4-methoxy-2-nitrobenzene $\mathbf{2 b}$ $(0.80 \mathrm{~g}, 3.25 \mathrm{mmol})$, after recrystalisation, gave diethyl 2-fluoro-2-(4-methoxy2-nitrobenzyl)malonate 3b (0.63 g, 56\%) as white crystals; mp $66-67{ }^{\circ} \mathrm{C}$; $\left([\mathrm{MH}]^{+}\right.$, 322.1160. $\mathrm{C}_{15} \mathrm{H}_{18} \mathrm{NO}_{7} \mathrm{~F}$ requires $[\mathrm{MH}]^{+}$, 344.1146); $v_{\max }\left(\right.$ neat, $\mathrm{cm}^{-1}$ ) 2984, 1743, $1534,1295.9,1240,1212,1195,1046 ;{ }^{1} \mathrm{H}$ NMR $\delta 7.39\left(1 \mathrm{H}, \mathrm{d},{ }^{4} J_{\mathrm{HH}} 2.5, \mathrm{H}-3\right), 7.34$ $\left(1 \mathrm{H}, \mathrm{d},{ }^{3} J_{\mathrm{HH}} 8.6, \mathrm{H}-6\right), 7.06\left(1 \mathrm{H}, \mathrm{dd},{ }^{3} J_{\mathrm{HH}}\right.$ $\left.8.6,{ }^{4} J_{\mathrm{HH}} 2.6, \mathrm{H}-5\right), 4.29-4.18(4 \mathrm{H}, \mathrm{m}$, $\left.\mathrm{OCH}_{2}\right), 3.87\left(2 \mathrm{H}, \mathrm{d},{ }^{3} J_{\mathrm{HF}} 22.9, \mathrm{CF}-\mathrm{CH}_{2}\right)$, $3.85\left(3 \mathrm{H}, \mathrm{s}, \mathrm{OCH}_{3}\right), 1.26\left(6 \mathrm{H}, \mathrm{t},{ }^{3} \mathrm{~J}_{\mathrm{HH}} 7.1\right.$, $\left.\mathrm{CH}_{3}\right) ;{ }^{19} \mathrm{~F}$ NMR $\delta-164.25\left(\mathrm{t},{ }^{3} \mathrm{~J}_{\mathrm{HF}} 23.6\right) ;{ }^{13} \mathrm{C}$ NMR $\delta 165.51\left(\mathrm{~d},{ }^{2} J_{\mathrm{CF}} 25.6, \mathrm{C}=\mathrm{O}\right), 159.45$ (s, C-OMe), 151.21 (s, C-NO $)_{2}, 134.26$ (d, $\left.{ }^{3} J_{\mathrm{CF}} 2.3, \mathrm{C}-1\right), 119.34(\mathrm{~s}, \mathrm{Ar}), 119.12(\mathrm{~s}$, Ar), 110.01 (s, Ar), 94.00 (d, ${ }^{1} J_{\mathrm{CF}} 200.8$, $\mathrm{C}-\mathrm{F}), 63.05\left(\mathrm{~s}, \mathrm{OCH}_{2}\right), 55.94\left(\mathrm{~s}, \mathrm{OCH}_{3}\right)$, $34.83\left(\mathrm{~d},{ }^{2} J_{\mathrm{CF}} 20.3, \mathrm{CF}_{-} \mathrm{CH}_{2}\right), 14.00(\mathrm{~s}$, $\left.\mathrm{CH}_{3}\right) ; m / z$ (ASAP) $344.1\left([\mathrm{MH}]^{+}, 100 \%\right.$ ), 270.1 (33), 224.0 (67), 178.0 (63).

\subsubsection{Diethyl 2-fluoro-2-(2-fluoro-6- nitrobenzyl)malonate (3d)}

Sodium hydride $(0.13 \mathrm{~g}, 5.56 \mathrm{mmol})$, 1 (0.80 g, $4.49 \mathrm{mmol})$ and 2-fluoro-6-nitrobenzyl bromide 2d (1.00 g, 4.27 mmol), after recrystallization, gave diethyl 2-fluoro-2-(2-fluoro-6-nitrobenzyl) malonate $3 d(1.25 \mathrm{~g}, 88 \%)$ as a yellow solid; mp. 49-50 ${ }^{\circ} \mathrm{C}$; $\left([\mathrm{MH}]^{+}, 332.0953\right.$. $\mathrm{C}_{14} \mathrm{H}_{15} \mathrm{NO}_{6} \mathrm{~F}_{2}$ requires $\left.[\mathrm{MH}]^{+}, 332.0946\right)$; $v_{\max }\left(\right.$ neat, $\left.\mathrm{cm}^{-1}\right) 2997,1746,1533,1363$, $1252,1173,1075,1055 ;{ }^{1} \mathrm{H}$ NMR $\delta 7.75$ $\left(1 \mathrm{H}, \mathrm{m},{ }^{3} \mathrm{~J}_{\mathrm{HH}} 8.2, \mathrm{H}-3\right), 7.44\left(1 \mathrm{H}, \mathrm{td},{ }^{3} J_{\mathrm{HH}}\right.$ $\left.8.3,{ }^{4} \mathrm{~J}_{\mathrm{HF}} 5.5, \mathrm{H}-4\right), 7.37-7.31(1 \mathrm{H}, \mathrm{m}, \mathrm{H}-5)$, 4.34-4.22 (4H, m, $\left.\mathrm{OCH}_{2}\right), 4.06(2 \mathrm{H}, \mathrm{dd}$, $\left.{ }^{3} J_{\mathrm{HF}} 20.6,{ }^{4} J_{\mathrm{HF}} 1.8, \mathrm{CF}_{-} \mathrm{CH}_{2}\right), 1.29(6 \mathrm{H}, \mathrm{t}$, $\left.{ }^{3} J_{\mathrm{HF}}^{\mathrm{HF}} 7.1, \mathrm{CH}_{3}\right) ;{ }^{\mathrm{HF}} \mathrm{F} \mathrm{NMR} \delta-110.14(1 \mathrm{~F}, \mathrm{~m}$, Ar-F), -164.80 (1F, t, $\left.{ }^{3} J_{\mathrm{HF}} 20.6, \mathrm{CH}_{2}-\mathrm{CF}\right)$; ${ }^{13} \mathrm{C}$ NMR $\delta 165.39\left(\mathrm{~d},{ }^{\mathrm{HF}} J_{\mathrm{CF}} 25.6, \mathrm{C}=\mathrm{O}\right)$, $161.63\left(\mathrm{~d},{ }^{1} J_{\mathrm{CF}} 250.9\right.$, Ar-F), 151.38 (d, $\left.{ }^{3} J_{\mathrm{CF}} 3.9 \mathrm{~Hz}, \mathrm{C}-\mathrm{NO}_{2}\right), 129.68\left(\mathrm{~d},{ }^{3} J_{\mathrm{CF}} 9.5\right.$, C-4), 121.02 (d, $\left.{ }^{4} J_{\mathrm{CF}} 3.3, \mathrm{C}-3\right), 120.20$ (d, $\left.{ }^{2} J_{\mathrm{CF}} 24.2, \mathrm{C}-5\right), 116.37\left(\mathrm{~d},{ }^{2} J_{\mathrm{CF}} 19.9, \mathrm{C}-1\right)$, $92.69\left(\mathrm{~d},{ }^{1} \mathrm{~J}_{\mathrm{CF}} 201.6, \mathrm{CF}\right), 63.16\left(\mathrm{~s}, \mathrm{OCH}_{2}\right)$, $28.25\left(\mathrm{dd},{ }^{2} J_{\mathrm{CF}} 21.4,{ }^{3} J_{\mathrm{CF}} 3.1, \mathrm{CF}-\mathrm{CH}_{2}\right)$, $13.95\left(\mathrm{~s}, \mathrm{CH}_{3}\right) ; \mathrm{m} / \mathrm{z}(\mathrm{ESI}) 332.1\left([\mathrm{MH}]^{+}\right.$, $100 \%)$.

\subsubsection{Diethyl 2-(5-chloro-2-nitro- benzyl)-2-fluoromalonate (3e) and Tetraethyl 2-(5-chloro-2-nitro- phenyl)-1-fluoropropane-1,1,3,3- tetracarboxylate (5)}

Sodium hydride $(0.12 \mathrm{~g}, 4.91 \mathrm{mmol})$ was washed with hexane $(2 \times 50 \mathrm{~mL})$ and added to dry DMF (15 mL). 1 (0.69 g, 3.89 $\mathrm{mmol})$ in dry DMF $(10 \mathrm{~mL})$ was added drop wise, followed by 5-chloro-2-nitrobenzyl methanesulfonate $4(1.00 \mathrm{~g}, 3.78 \mathrm{mmol})$ in DMF (15 mL). After $5 \mathrm{~h}$, more $1(0.17 \mathrm{~g}$,
$0.95 \mathrm{mmol})$ was added with $\mathrm{NaH}(0.09 \mathrm{~g}$, $3.75 \mathrm{mmol})$, then after $20 \mathrm{~h}$ the solution was added to ice/water $(150 \mathrm{~mL})$, extracted with diethyl ether $(3 \times 50 \mathrm{~mL})$, washed with water and brine, dried $\left(\mathrm{MgSO}_{4}\right)$, concentrated and purified by column chromatography using hexane, ethyl acetate as elutant to give diethyl 2-(5-chloro-2-nitrobenzyl)-2-fluoromalonate $3 \mathrm{e}(0.44 \mathrm{~g}$, $33 \%)$ as yellow crystals; mp $52-53{ }^{\circ} \mathrm{C}$; $\left([\mathrm{MH}]^{+}, \quad 348.0648 . \quad \mathrm{C}_{14} \mathrm{H}_{15} \mathrm{NO}_{6} \mathrm{~F}^{35} \mathrm{Cl}\right.$ requires $[\mathrm{MH}]^{+}, 348.0650$ ); $v$ (neat, $\mathrm{cm}^{-1}$ ) 2992, 1746, 1524, 1352, 1276, 1192, 1044; ${ }^{1} \mathrm{H}$ NMR $\delta 7.87\left(1 \mathrm{H}, \mathrm{d},{ }^{3} J_{\mathrm{HH}} 8.6, \mathrm{H}-3\right), 7.44$ (1H, s, H-6), $7.41\left(\mathrm{dd},{ }^{3} J_{\mathrm{HH}} 8.6,{ }^{4} J_{\mathrm{HH}} 2.3\right.$, $\mathrm{H}-4), 4.34-4.19$ (4H, m, OCH $), 3.94(2 \mathrm{H}$, $\left.\mathrm{d},{ }^{3} \mathrm{~J}_{\mathrm{HF}} 22.8, \mathrm{CH}_{2}-\mathrm{CF}\right), 1.27\left(6 \mathrm{H}, \mathrm{t},{ }^{3} \mathrm{~J}_{\mathrm{HH}} 7.1\right.$, $\left.\mathrm{CH}_{3}\right) ;{ }^{\mathrm{HF}} \mathrm{F}$ NMR $\delta-164.12\left(\mathrm{t},{ }^{3} \mathrm{~J}_{\mathrm{HF}} 22.8\right) ;{ }^{13} \mathrm{C}$ NMR $\delta 165.19\left(\mathrm{~d},{ }^{2} J_{\mathrm{CF}} 25.4, \mathrm{C}=\mathrm{O}\right), 148.88$ (s, C-NO $), 139.04$ (s, C-Cl), 133.36 (s, Ar), 129.84 (s, Ar), 128.99 (s, Ar), 126.54 (s, Ar), 93.55 (d, ${ }^{1} J_{\mathrm{CF}}$ 202.3, C-F), 63.27 (s, $\left.\mathrm{OCH}_{2}\right), 35.29\left(\mathrm{~d},{ }^{2} J_{\mathrm{CF}} 20.4, \mathrm{CF}-\mathrm{CH}_{2}\right), 13.98$ $\left(\mathrm{s}, \mathrm{CH}_{3}\right) ; \mathrm{m} / \mathrm{z}$ (ASAP) $348.1\left([\mathrm{MH}]^{+}\right)$, 100\%), 274.0 (14), 228.0 (20); and, tetraethyl 2-(5-chloro-2-nitrophenyl)-1-fluoropropane-1,1,3,3-tetracarboxylate $5(0.73$ $\mathrm{g}, 55 \%)$ as an orange oily solid; $\mathrm{mp}$ 40-41 ${ }^{\circ} \mathrm{C}$; $\left([\mathrm{MH}]^{+}, 506.1210 . \mathrm{C}_{21} \mathrm{H}_{25} \mathrm{NO}_{10} \mathrm{~F}^{35} \mathrm{Cl} \mathrm{re}-\right.$ quires $\left.[\mathrm{MH}]^{+}, 506.1229\right) ; v_{\text {max }}$ (neat, $\mathrm{cm}^{-1}$ ) 2982, 1752, 1532, 1231, 1147, 1095, 1047: ${ }^{1} \mathrm{H} N M R \delta 7.83\left(1 \mathrm{H}, \mathrm{d},{ }^{3} J_{\mathrm{HH}} 8.7, \mathrm{H}-3\right), 7.64$ $7.58(1 \mathrm{H}, \mathrm{m}, \mathrm{H}-8), 7.39\left(1 \mathrm{H}, \mathrm{dd},{ }^{3} J_{\mathrm{HH}} 8.7\right.$, $\left.{ }^{4} J_{\mathrm{HH}} 2.2, \mathrm{H}-4\right), 5.63\left(1 \mathrm{H}, \mathrm{dd},{ }^{3} J_{\mathrm{HF}} 27.4,{ }^{3} J_{\mathrm{HH}}\right.$ 9.8, $\mathrm{CH}-\mathrm{CF}), 4.37-3.76\left(9 \mathrm{H}, \mathrm{m}, \mathrm{OCH}_{2}\right.$, $\mathrm{CH}-\mathrm{C}=\mathrm{O}), 1.33\left(3 \mathrm{H}, \mathrm{t},{ }^{3} \mathrm{~J}_{\mathrm{HH}} 7.2, \mathrm{CH}_{3}\right), 1.27$ $\left(3 \mathrm{H}, \mathrm{t},{ }^{3} J_{\mathrm{HH}} 7.1, \mathrm{CH}_{3}\right), 1.06\left(3 \mathrm{H}, \mathrm{t},{ }^{3} J_{\mathrm{HH}} 7.1\right.$, $\left.\mathrm{CH}_{3}\right), 1.00\left(3 \mathrm{H}, \mathrm{t},{ }^{3}{ }^{3}{ }_{\mathrm{HH}} 7.1, \mathrm{CH}_{3}\right) ;{ }^{19} \mathrm{~F}$ NMR $\delta-168.93\left(\mathrm{~d},{ }^{3} J_{\mathrm{HF}} 27.4\right) ;{ }^{13} \mathrm{C}$ NMR $\delta 166.89$ (s, C=O), 166.35 (s, C=O), $164.48\left(\mathrm{~d},{ }^{2} J_{\mathrm{CF}}\right.$ 24.5, FC-C $=\mathrm{O}), 164.22$ (d, ${ }^{2} J_{\mathrm{CF}} 23.9$, FC$\mathrm{C}=\mathrm{O}), 148.96\left(\mathrm{~s}, \mathrm{C}-\mathrm{NO}_{2}\right), 139.00(\mathrm{~s}, \mathrm{C}-\mathrm{Cl})$, $132.56\left(\mathrm{~d},{ }^{4} J_{\mathrm{CF}} 1.3, \mathrm{C}-6\right), 130.95\left(\mathrm{~d},{ }^{3} J_{\mathrm{CF}}\right.$ 6.3, C-1), 129.19 (s, C-4), 126.37 (s, C-3), $94.91\left(\mathrm{~d},{ }^{1} J_{\mathrm{CF}} 207.2, \mathrm{C}-\mathrm{F}\right), 63.65\left(\mathrm{~s}, \mathrm{OCH}_{2}\right)$, $63.23\left(\mathrm{~s}, \mathrm{OCH}_{2}\right), 62.49\left(\mathrm{~s}, \mathrm{OCH}_{2}\right), 62.12(\mathrm{~s}$, $\left.\mathrm{OCH}_{2}\right), 52.85\left(\mathrm{~d},{ }^{3} J_{\mathrm{CF}} 5.7, \underline{\mathrm{CH}}-\mathrm{C}=\mathrm{O}\right), 40.75$ $\left(\mathrm{d},{ }^{2} J_{\mathrm{CF}} 18.6, \mathrm{CF}-\mathrm{CH}\right), 14.02\left(\mathrm{~s}, \mathrm{CH}_{3}\right)$, $13.88\left(\mathrm{~s}, \mathrm{CH}_{3}\right), 13.72\left(\mathrm{~s}, \mathrm{CH}_{3}\right), 13.67$ (s, $\left.\mathrm{CH}_{3}\right) ; \mathrm{m} / z$ (ASAP) $506.1\left([\mathrm{MH}]^{+}, 100 \%\right)$, 460.1 (85), 414.0 (72).

\subsection{Synthesis of Fluorotetra- hydroquinolines 6 by Reduction- Cyclisation}

4.3.1 Method A: Hydrogenation over $\mathrm{Pd} / \mathrm{C}$

Diester 3 and $\mathrm{Pd} / \mathrm{C}$ in acetic acid were placed in a Parr hydrogenator and pressurised (40 psi) with hydrogen. After stirring for $1 \mathrm{~h}$ the solution was filtered through celite, concentrated, DCM $(50 \mathrm{~mL})$ added, washed with sodium bicarbonate $(3 \times 50$ $\mathrm{mL})$, dried $\left(\mathrm{MgSO}_{4}\right)$ and concentrated to give the desired fluorotetrahydroquinoline 6 which was purified by recrystallization if required.

\subsubsection{Method B: Sodium Dithionite}

Sodium bicarbonate was added slowly to a solution of diester 3 in THF and water. Sodium dithionite was added portion wise over 10 minutes with vigorous stirring. After 30 minutes, brine $(8 \mathrm{~mL})$ and ethyl acetate $(8 \mathrm{~mL})$ were added, the organic layer separated and washed with sodium bicarbonate $(3 \times 50 \mathrm{~mL})$ and brine $(3 \times 50 \mathrm{~mL})$, concentrated and the residue purified by column chromatography using hexane:ethyl acetate $(9: 1)$ as elutant to give the desired fluorotetrahydroquinoline product 6.

\subsubsection{Ethyl 3-fluoro-2-oxo-1,2,3,4- tetrahydroquinoline-3-carboxylate (6a)}

Method A: Diethyl 2-fluoro-2-(2-nitrobenzyl)malonate $\mathbf{3 a}(1.25 \mathrm{~g}, 4 \mathrm{mmol})$ and $\mathrm{Pd} / \mathrm{C}(0.43 \mathrm{~g}, 5 \mathrm{~mol} \% \mathrm{Pd})$ in acetic acid (40 $\mathrm{mL}$ ) gave ethyl 3-fluoro-2-oxo-1,2,3,4-tetrahydroquinoline-3-carboxylate $\mathbf{6 a}(0.73$ $\mathrm{g}, 78 \%$ ) as a white powder; $\mathrm{mp} 84-85^{\circ} \mathrm{C}$; $\left([\mathrm{MH}]^{+}, 238.0862 . \mathrm{C}_{12} \mathrm{H}_{12} \mathrm{FNO}_{3}\right.$ requires $\left.[\mathrm{MH}]^{+}, 238.0879\right) ; v_{\text {max }}$ (neat, $\mathrm{cm}^{-1}$ ) 3215, 3096, 2992, 1752, 1688, 1493, 1200, 1131; ${ }^{1} \mathrm{H}$ NMR $\delta 9.03(1 \mathrm{H}, \mathrm{s}, \mathrm{NH}), 7.24(1 \mathrm{H}, \mathrm{t}$, $\left.{ }^{3} J_{\mathrm{HH}} 8, \mathrm{Ar}-\mathrm{H}\right), 7.18\left(1 \mathrm{H}, \mathrm{d},{ }^{3} J_{\mathrm{HH}} 7.4, \mathrm{Ar}-\mathrm{H}\right)$, $7.06\left(1 \mathrm{H}, \mathrm{t},{ }^{3} J_{\mathrm{HH}} 7.0, \mathrm{Ar}-\mathrm{H}\right), 6.90(1 \mathrm{H}, \mathrm{d}$, $\left.{ }^{3} J_{\mathrm{HH}} 7.9, \mathrm{Ar}-\mathrm{H}\right), 4.35-4.21\left(2 \mathrm{H}, \mathrm{m}, \mathrm{OCH}_{2}\right)$, 3.68-3.40 (2H, m, CF-CH $), 1.23(3 \mathrm{H}, \mathrm{t}$, $\left.{ }^{3} J_{\mathrm{HH}} 7.1, \mathrm{CH}_{3}\right) ;{ }^{19} \mathrm{~F}$ NMR $\delta-165.17(\mathrm{dd}$, $\left.{ }^{3} J_{\mathrm{HF}}^{\mathrm{HH}} 23.4,{ }^{3} J_{\mathrm{HF}}^{3} 15.4\right) ;{ }^{13} \mathrm{C} \mathrm{NMR} \delta 166.57$ $\left(\mathrm{d},{ }^{2} J_{\mathrm{CF}} 25.8, \mathrm{C}=\mathrm{O}\right), 164.05\left(\mathrm{~d},{ }^{2} J_{\mathrm{CF}} 22.8\right.$, $\mathrm{C}=\mathrm{O}$ ), 135.52 (s, C-NH), 128.77 (s, Ar), 128.58 (s, Ar), 124.22 (s, Ar), 118.98 (d, $\left.{ }^{3} J_{\mathrm{CF}} 6.5, \mathrm{C}-4 \mathrm{a}\right), 116.00(\mathrm{~s}, \mathrm{Ar}), 90.32$ (d, $\left.{ }^{1} J_{\mathrm{CF}}^{\mathrm{CF}} 195.1, \mathrm{C}-\mathrm{F}\right), 62.93\left(\mathrm{~s}, \mathrm{OCH}_{2}\right) 35.65$ $\left(\mathrm{d},{ }^{2} J_{\mathrm{CF}} 23.8, \mathrm{CF}-\mathrm{CH}_{2}\right), 14.03\left(\mathrm{~s}, \mathrm{CH}_{3}\right) ; \mathrm{m} / \mathrm{z}$ (ASAP) $238.1\left([\mathrm{MH}]^{+}, 23 \%\right), 210.1$ (40), 164.0 (100).

Method B: Sodium bicarbonate (1.51 g, $17.98 \mathrm{mmol}$ ), diethyl 2-fluoro-2-(2-nitrobenzyl)malonate 3a $(1.00 \mathrm{~g}, 3.19 \mathrm{mmol})$ in THF $(8 \mathrm{~mL})$ and water $(8 \mathrm{~mL})$ and sodium dithionite $(2.85 \mathrm{~g}, 16.38 \mathrm{mmol})$ gave ethyl 3-fluoro-2-oxo-1,2,3,4-tetrahydroquinoline-3-carboxylate 6a (0.43 g, 57\%) as a white powder; physical and spectral data as above.

\subsubsection{Ethyl 7-cyano-3-fluoro-2- oxo-1,2,3,4-tetrahydroquinoline-3- carboxylate (6b)}

Method B: Sodium bicarbonate (1.51 g, $17.98 \mathrm{mmol})$, diethyl 2-(4-cyano-2-nitrobenzyl)-2-fluoromalonate 3b $(0.5 \mathrm{~g}, 1.48 \mathrm{mmol})$ in THF $(8 \mathrm{~mL})$ and water $(8 \mathrm{~mL})$ and sodium dithionite $(2.85 \mathrm{~g}, 16.38 \mathrm{mmol})$, gave ethyl 7-cyano-3-fluoro-2-oxo-1,2,3,4-tetrahydroquinoline-3-carboxylate $6 \mathbf{b}(0.30 \mathrm{~g}, 76 \%)$ as an orange solid; $\mathrm{mp} 137-138^{\circ} \mathrm{C} ;\left([\mathrm{MH}]^{+}\right.$, 263.0848. $\mathrm{C}_{13} \mathrm{H}_{11} \mathrm{~N}_{2} \mathrm{O}_{3} \mathrm{~F}$ requires $[\mathrm{MH}]^{+}$, 263.0832); $v_{\max }\left(\right.$ neat, $\left.\mathrm{cm}^{-1}\right)$ 2981, 2922, 2226, 1758, 1695, 1257, 1205, 1103; ${ }^{1} \mathrm{H}$ 
NMR $\delta 9.23(1 \mathrm{H}, \mathrm{s}, \mathrm{NH}), 7.38\left(1 \mathrm{H}, \mathrm{d},{ }^{3} J_{\mathrm{HH}}\right.$ 8.3, H-6), $7.32\left(1 \mathrm{H}, \mathrm{d},{ }^{3} J_{\mathrm{HH}} 7.8, \mathrm{H}-5\right), 7.20$ $(1 \mathrm{H}, \mathrm{s}, \mathrm{H}-8), 4.38-4.28\left(2 \mathrm{H}, \mathrm{m}, \mathrm{OCH}_{2}\right)$, 3.79-3.42 (2H, m, CF-CH $), 1.28(3 \mathrm{H}, \mathrm{t}$, $\left.{ }^{3} J_{\mathrm{HH}} 7.1, \mathrm{CH}_{3}\right) ;{ }^{19} \mathrm{~F}$ NMR $\delta-165.38(\mathrm{dd}$, $\left.{ }^{3} J_{\mathrm{HF}} 24.9,{ }^{3} J_{\mathrm{HF}} 15.3\right) ;{ }^{13} \mathrm{C} \mathrm{NMR} \delta 165.94$ $\left(\mathrm{d},{ }^{2} J_{\mathrm{CF}} 25.4, \mathrm{C}=\mathrm{O}\right), 163.87\left(\mathrm{~d},{ }^{2} J_{\mathrm{CF}} 22.3\right.$, $\mathrm{C}=\mathrm{O}$ ), 136.47 (s, C-NHR), 129.70 (s, Ar), 127.86 (s, Ar), 124.48 (d, ${ }^{3} J_{\mathrm{CF}} 6.2$, C-4a), 119.07 (s, Ar), 117.96 (s, C-7), 112.53 (s, CN), 89.51 (d, $\left.{ }^{1} J_{\mathrm{CF}} 196.3, \mathrm{CF}\right), 63.41$ $\left(\mathrm{OCH}_{2}\right), 35.68\left(\mathrm{~d},{ }^{2} J_{\mathrm{CF}} 24.1, \mathrm{CF}-\mathrm{CH}_{2}\right)$, $14.06\left(\mathrm{~s}, \mathrm{CH}_{3}\right) ; \mathrm{m} / \mathrm{z}(\mathrm{ASAP}) 263.1\left([\mathrm{MH}]^{+}\right.$, 100\%), 243.1 (43), 189.0 (27).

\subsubsection{Ethyl 3-fluoro-7-methoxy -2-oxo-1,2,3,4-tetrahydroquino- line-3-carboxylate (6c)}

Method A: Diethyl 2-fluoro-2-(4-methoxy-2-nitrobenzyl)malonate $3 c(0.50 \mathrm{~g}$, $1.46 \mathrm{mmol})$ and $\mathrm{Pd} / \mathrm{C}(0.16 \mathrm{~g}, 5 \mathrm{~mol} \% \mathrm{Pd})$ in acetic acid $(20 \mathrm{~mL})$ after column chromatography and recrystallization, gave ethyl 3-fluoro-7-methoxy-2-oxo-1,2,3,4-tetrahydroquinoline-3-carboxylate $\mathbf{6 c}(0.26$ g, 67\%) as white needles; mp $111-112{ }^{\circ} \mathrm{C}$; $\left([\mathrm{MH}]^{+}, 268.0984 . \mathrm{C}_{13} \mathrm{H}_{15} \mathrm{FNO}_{4}\right.$ requires $\left.[\mathrm{MH}]^{+}, 268.0985\right) ; v_{\max }\left(\right.$ neat, $\left.\mathrm{cm}^{-1}\right) 2984$, 1761, 1693, 1288, 1255, 1068; ${ }^{1} \mathrm{H}$ NMR $\delta 7.84(1 \mathrm{H}, \mathrm{s}, \mathrm{NH}), 7.08\left(1 \mathrm{H}, \mathrm{d},{ }^{3} \mathrm{~J}_{\mathrm{HH}} 8.4\right.$, Ar-H), 6.59 (1H, d, $\left.{ }^{3} J_{\mathrm{HH}} 8.4, \mathrm{Ar}-\mathrm{H}\right), 6.36$ (1H, s, Ar-H), 4.33-4.22 (2H, m, OCH $)$, $3.79\left(3 \mathrm{H}, \mathrm{s}, \mathrm{OCH}_{3}\right), 3.63-3.29(2 \mathrm{H}, \mathrm{m}$, $\left.\mathrm{CF}_{-} \mathrm{CH}_{2}\right), 1.24\left(3 \mathrm{H}, \mathrm{t},{ }^{3} \mathrm{~J}_{\mathrm{HH}} 7.1, \mathrm{CH}_{3}\right) ;{ }^{19} \mathrm{~F}$ NMR $\delta-165.75\left(\mathrm{dd},{ }^{3} J_{\mathrm{HF}} 22.3,{ }^{3} J_{\mathrm{HF}} 15.0\right)$; ${ }^{13} \mathrm{C}$ NMR $\delta 166.59\left(\mathrm{~d},{ }^{2} J_{\mathrm{CF}} 25.8, \mathrm{C}=\mathrm{O}\right)$, $163.95\left(\mathrm{~d},{ }^{2} J_{\mathrm{CF}} 22.8, \mathrm{C}=\mathrm{O}\right), 159.97$ (s, C-OMe), 136.44 (s, C-NHR), 129.70 (s, Ar), $110.92\left(\mathrm{~d},{ }^{3} J_{\mathrm{CF}}\right.$ 7.0, Ar), 109.41 (s, Ar), 102.03 (s, Ar), 90.42 (d, $\left.{ }^{1} J_{\mathrm{CF}} 195.5, \mathrm{C}-\mathrm{F}\right)$, $62.90\left(\mathrm{~s}, \mathrm{OCH}_{2}\right), 55.63\left(\mathrm{~s}, \mathrm{OCH}_{3}\right), 34.97$ $\left(\mathrm{d},{ }^{2} \mathrm{~J}_{\mathrm{CF}} 23.8, \mathrm{CF}-\mathrm{CH}_{2}\right), 14.06\left(\mathrm{~s}, \mathrm{CH}_{3}\right) ; \mathrm{m} / \mathrm{z}$ (ASAP) 268.1 ([MH $\left.]^{+}, 100 \%\right), 248.1$ (28), 194.1 (39).
4.3.6 Ethyl 3,5-difluoro-2-oxo-1,2,3,4tetrahydroquinoline-3-carboxylate (6d)

Method A: Diethyl 2-fluoro-2-(2fluoro-6-nitrobenzyl)malonate 3d (1.00 g, $3.02 \mathrm{mmol})$ and $\mathrm{Pd} / \mathrm{C}(0.32 \mathrm{~g}, 5 \mathrm{~mol} \%$ $\mathrm{Pd})$ in acetic acid $(30 \mathrm{~mL})$ after $3.5 \mathrm{~h}$ gave ethyl 3,5-difluoro-2-oxo-1,2,3,4-tetrahydroquinoline-3-carboxylate 6d (0.67 g, $87 \%)$ as a dark red viscous oil; $\left([\mathrm{MH}]^{+}\right.$, 256.0785. $\mathrm{C}_{12} \mathrm{H}_{11} \mathrm{~F}_{2} \mathrm{NO}_{3}$ requires $[\mathrm{MH}]^{+}$, 256.0785); v (neat, $\mathrm{cm}^{-1}$ ) 3232, 2984, 1691, 1600, 1486, 1273, 1073; ' ${ }^{\max } \mathrm{NMR}$ $\left(600 \mathrm{MHz}, \mathrm{DMSO}-d_{6}\right) \delta 11.18(1 \mathrm{H}, \mathrm{s}$, $\mathrm{NH}), 7.28\left(1 \mathrm{H}, \mathrm{q},{ }^{3} J_{\mathrm{HH}} 8.0, \mathrm{H}-6\right), 6.91(1 \mathrm{H}$, $\left.\mathrm{t},{ }^{3} J_{\mathrm{HH}} 8.8, \mathrm{H}-7\right), 6.81\left(1 \mathrm{H}, \mathrm{d},{ }^{3} J_{\mathrm{HH}} 8.0, \mathrm{H}-8\right)$, 4.29-4.16 $\left(2 \mathrm{H}, \mathrm{m}, \mathrm{OCH}_{2}\right), 3.68-3.46(2 \mathrm{H}$, $\left.\mathrm{m}, \mathrm{CF}-\mathrm{CH}_{2}\right), 1.16\left(3 \mathrm{H}, \mathrm{t},{ }^{3} \mathrm{~J}_{\mathrm{HH}} 7.1, \mathrm{CH}_{3}\right)$; ${ }^{19} \mathrm{~F}$ NMR (564 MHz, DMSO-d $) \delta-119.34$ --119.44 (1F, m, Ar-F), -163.80 (1F, dd, $\left.{ }^{3} J_{\mathrm{HF}} 24.4,{ }^{3} J_{\mathrm{HF}} 20.6, \mathrm{C}-\mathrm{F}\right) ;{ }^{13} \mathrm{C}$ NMR $(151$ $\left.\mathrm{MHz}, \mathrm{DMSO}-d_{6}\right) \delta 166.05\left(\mathrm{~d},{ }^{2} J_{\mathrm{CF}} 25.7\right.$, $\mathrm{C}=\mathrm{O}), 161.92\left(\mathrm{~d},{ }^{2} J_{\mathrm{CF}} 22.7, \mathrm{C}=\mathrm{O}\right), 159.73$ $\left(\mathrm{d},{ }^{1} J_{\mathrm{CF}} 243.4, \mathrm{Ar}-\mathrm{F}\right), 138.08\left(\mathrm{~d},{ }^{3} J_{\mathrm{CF}} 6.8\right.$, C-8a), 129.35 (d, $\left.{ }^{3} J_{\mathrm{CF}} 9.5, \mathrm{C}-7\right), 111.49$ (d, $\left.{ }^{4} J_{\mathrm{CF}} 2.8, \mathrm{C}-8\right), 109.80\left(\mathrm{~d},{ }^{2} J_{\mathrm{CF}} 21.3, \mathrm{C}-6\right)$, $106.46\left(\mathrm{dd},{ }^{2} J_{\mathrm{CF}} 21.2,{ }^{3} J_{\mathrm{CF}} 5.7, \mathrm{C}-4 \mathrm{a}\right), 89.56$ $\left(\mathrm{d},{ }^{1} J_{\mathrm{CF}} 192.0, \underline{\mathrm{CF}}-\mathrm{CH}_{2}\right), 62.27\left(\mathrm{~s}, \mathrm{OCH}_{2}\right)$, $28.01{ }^{\mathrm{CF}}\left(\mathrm{dd},{ }^{2} \mathrm{~J}_{\mathrm{CF}} 24.4,{ }^{3} \mathrm{~J}_{\mathrm{CF}} 3.0, \mathrm{CF}-\mathrm{CH}_{2}\right)$, $13.73\left(\mathrm{~s}, \mathrm{CH}_{3}\right) ; \mathrm{m} / \mathrm{z}(\mathrm{ASAP}) 256.1\left([\mathrm{MH}]^{+}\right.$, $34 \%), 236.1$ (100).

\section{Acknowledgements}

We thank the Durham University M. Chem. programme for funding.

Received: April 1, 2014

[1] Fluorine in pharmaceuticals: a) K. Muller, C. Faeh, F. Diedrich, Science 2007, 317, 1881; b) 'Fluorine in Medicinal Chemistry and Chemical Biology', Ed. I. Ojima, Wiley-Blackwell: Oxford, 2009; c) S. Purser, P. R. Moore, S. Swallow, V. Gouverneur, Chem. Soc. Rev. 2008, 37, 320; d) C. Isanbor, D. O'Hagan, J. Fluorine Chem. 2006, 127, 303; e) K. L. Kirk, J. Fluorine Chem. 2006, 127, 1013.

[2] a) G. Sandford, J. Fluorine Chem. 2007, 128, 90; b) C. B. McPake, G. Sandford, Org. Proc. Res. Dev. 2012, 16, 844.
[3] R. D. Chambers, J. Hutchinson, J. Thomson, J. Fluorine Chem. 1996, 78, 165.

[4] a) R. D. Chambers, J. Hutchinson, J. Fluorine Chem. 1998, 92, 45; b) R. D. Chambers, M. A. Fox, D. Holling, T. Nakano, T. Okazoe, G. Sandford, Chem. Eng. Tech. 2005, 28, 344.

[5] Use of dicarbonyl systems in organic synthesis: a) S. Warren, 'Designing Organic Syntheses: The Synthon Approach', John Wiley and Sons, New York, 1978; b) S. Warren, P. Wyatt, 'Organic Synthesis: The Disconnection Approach', 2nd ed., John Wiley and Sons, Chichester, 2008; c) S. Warren, P. Wyatt, 'Organic Synthesis: Strategy and Control', 2nd ed., John Wiley and Sons, Chichester, 2007; d) E. J. Corey, X.M. Cheng, 'The logic of chemical synthesis', Wiley-Interscience, New York, 1995.

[6] Diethyl fluoromalonate in synthesis: a) T. A. Miller, D. L. Sloman, M. G. Stanton, K. J. Wilson, D. J. Witter, WO2007087129, 2007; b) J. Close, R. W. Heidebrecht, S. Kattar, T. A. Miller, D. Sloman, M. G. Stanton, P. Tempest, D. J. Witter, WO2007055941, 2007; c) J. L. Hubbs, D. M. Mampreian, J. L. Methot, T. A. Miller, K. M. Otte, P. Siliphaivanh, D. L. Sloman, M. G. Stanton, K. J. Wilson, D. J. Witter, WO2006115845, 2006; d) M. O. Polla, L. Tottie, C. Nordén, M. Linchoten, D. Müsil, S. Trumpp-Kallmeyer, I. R. Aukrust, R. Ringom, K. H. Holm, S. M. Neset, M. Sandberg, J. Thurmond, P. Yu, G. Hategan, H. Anderson, Bioorg. Med. Chem. 2004, 12, 1151; e) K. Kwon, S. M. Kim, D. Y. Kim, J. Fluorine Chem. 2009, 130, 759; f) H. Li, L. Zu, H. Xie, W. Wang, Synthesis 2009, 1525.

[7] D. Seebach, Angew. Chem. Intl. Ed. Engl. 1990, 29, 1320.

[8] a) S. Jaroch, H. Rehwinkel, P. Holscher, D. Sulzle, G. Burton, M. Hillmann, F. M. McDonald, H. Miklautz, Bioorg. Med. Chem. Lett. 2004, 14, 743; b) S. Jaroch, H. Rehwinkel, P. Holscher, D. Sulzle, M. Hillmann, G. Burton, F. M. McDonald, PCT Intl. Pat. Appl., WO 00/29381, 2000.

[9] A. C. Dikundwar, T. N. Guru Row, Cryst. Growth Des. 2012, 12, 1713.

[10] Fluorine gauche effect in amides: J. W. Banks, A. S. Batsanov, J. A. K. Howard, D. O'Hagan, H. S. Rzepa, S. Martin-Santamaria, J. Chem. Soc., Perkin Trans. 2 1999, 2409; D. O'Hagan, Chem. Soc. Rev. 2008, 37, 308.

[11] a) G. M. Sheldrick, Acta Cryst., Sect. A 2008, 64, 112; b) O. V. Dolomanov, L. J. Bourhis, R. J. Gildea, J. A. K. Howard, H. Puschmann, J. Appl. Cryst. 2009, 42, 339. 